\title{
INNOVACIÓN SOCIAL Y DISEÑO PARTICIPATIVO PARA LA TRANSFORMACIÓN SOSTENIBLE DE LA PLAYA DE JUANCHACO
}

\section{Entorno Ambiente}

Stephanía Silva Salazar

stephania62551@gmail.com

Laura Albarracín, Laura Sofía Duque, Juan

Fernando Lopera, Aura Lozano

Universidad ICESI

Semillero Espacio D+E+I

Cali - Valle del Cauca. 


\section{Resumen}

El proyecto aborda la problemática de contaminación por desechos plásticos en la - playa de Juanchaco, provocada por la ausencia de un sistema de gestión de basuras, las condiciones geográficas de la zona y el flujo de turistas que incrementa la cantidad de residuos. Estos factores han causado la acumulación de desechos a la intemperie, para afectar de manera negativa la dinámica ecosistémica, en detrimento de la calidad de vida y el desarrollo regional. Por esto, desde el diseño y la innovación social, se quiere intervenir la playa de Juanchaco de mano con la comunidad, para generar un impacto transformador en su calidad de vida. Se utilizó la etnografía como metodología para realizar un análisis cultural de los habitantes, partiendo de un estudio sistemático, consistente en la observación y participación con agentes de la comunidad. Así, se exploró y comprendió sobre aspectos de la forma de vida, cosmovisión y patrones de comportamiento de los habitantes. Esto para facilitar la ideación y materialización de una propuesta de diseño que armonice la situación descrita. Desde la identificación de oportunidades, se aplicaron herramientas de Design thinking que, en complemento con la observación y experimentación, permitieron entender y dar solución a las necesidades reales de los usuarios. Con la implementación del proyecto, se espera que la comunidad de Juanchaco se integre en el cuidado de la playa y llegue a resignificar los residuos como punto de partida para un proceso constructivo que, a su vez, permita la apropiación, conservación y transmisión de los aspectos sociales tradicionales que los caracteriza como comunidad.

\section{Introducción}

Colombia es el único país en Suramérica con acceso a dos océanos: el Pacífico y el Atlántico. Esto la convierte en un atractivo para inversión, por el fácil acceso de mercancía, la explotación y cuidado de los recursos oceánicos y otras ventajas, como es el caso del puerto de Buenaventura. Esta ciudad, además de ser motor de desarrollo económico, es parte del Parque Nacional Natural Uramba Bahía Málaga, zona en la que se conservan ecosistemas marinos y costeros, se articulan prácticas tradicionales orientadas al fortalecimiento de la dinámica cultural y organización social (Parques Nacionales Naturales de Colombia, 2019).

Sin embargo, los estudios afirman que en esta región se presentan las tasas más altas de pobreza multidimensional (33.3\%), lo que hace que sea menos atractiva para inversión estatal y, por el contrario, es marginada (Resultados de pobreza en 2018 por regiones y ciudades, 2019; Rodas, 2017). Esto ha generado escasez en los recursos básicos y un gran problema de salubridad con el mal manejo de desechos.

Esta situación afecta, también, a las zonas aledañas al puerto, pues las corrientes de mar y viento arrastran los desperdicios hacia otros territorios. Una de las playas más afectadas al noroeste de Buenaventura, es Juanchaco. La economía de este poblado, se centra en la extracción de madera, pesca artesanal, recolección de piangua y el turismo (Molina Moreira, 2017); así mismo, se enfrenta a una problemática por acumulación de desechos en su playa. Gran parte de isleños y turistas no hacen buena disposición de los residuos que generan, 
no se gestiona la recolección ni existe un proceso para el tratamiento de basuras, por lo cual la mayoría de éstas termina en la playa y el océano, para desmejorar las condiciones del ecosistema.

Frente a esto, se identificó la necesidad de generar una estrategia que impulse una transformación cultural, al partir de un análisis en tres niveles: artefactos, valores y supuestos. El primero representa el análisis de la parte visible de la actividad cultural; el segundo profundiza en la población, los artefactos y comportamiento de los habitantes; y el tercero analiza sus creencias fundamentales (Guevara Montesinos, 2017).

Ahora, para concretar esta transformación, se plantearon cinco fases importantes: a) determinar los aspectos sociales y tradicionales característicos de la comunidad con la que se va a trabajar; b) identificar las causas de las prácticas no sostenibles que persisten a pesar de las consecuencias; c) identificar impulsores potenciales (aliados) para el cambio -en el cumplimiento y búsqueda de impulsores se encuentra a EcoPAZifico, un colectivo con el deseo de liberar al océano de los desechos plásticos y contribuir al bienestar del planeta a partir de la implementación de sistemas de reciclaje, junto con programas educativos-; d) estimular iniciativas para sensibilizar a los habitantes de la isla, acerca de los beneficios medioambientales, económicos y sociales de hacer buena disposición de los residuos; e) gestionar la resistencia al cambio que muestra la población, al evidenciar las consecuencias de la problemática tanto para ellos como para los 1.5 millones de animales que mueren por consumo de plástico anualmente (Serrano, 2019).

Además, se desea generar transformación a partir de la innovación social, para lograr llegar a ideas nuevas sobre productos, servicios y modelos de negocio que satisfagan las necesidades de los nativos. Esto, no sólo para mitigar la problemática alrededor de los residuos, sino para impulsar el progreso socioeconómico de Juanchaco y considerar una visión sostenible a largo plazo, con una solución escalable y replicable en otros contextos que vivan situaciones similares.

\section{Metodología}

El proyecto consideró un componente social determinante; así entonces, la metodología tuvo en cuenta la participación de la comunidad, y en este sentido, abarcó tres etapas: exploración, definición y materialización, donde el trabajo colaborativo fue clave para alcanzar los objetivos. Esto se conjugó con la metodología de Design thinking, que permite entender y centrarse en el usuario y su contexto.

Exploración: esta etapa se enfocó en conocer y analizar la cosmovisión de la población de Juanchaco de forma integral, para identificar sus aspectos culturales y percepciones. Con la utilización de herramientas para la comprensión del ser, tales como mapas de empatía, entrevistas y encuestas que permitieron obtener información de primera mano. La observación, fotografía y trabajo con la comunidad facilitaron la comprensión y diagnóstico de la problemática. 
Definición: tras diagnosticar el problema y definir los objetivos para llegar a una solución, desde esta metodología participativa, se definieron requerimientos para generar un sistema de solución. Luego, utilizando herramientas de ideación, planteamiento de futuros y escenarios ideales, se llegó al planteamiento de posibles soluciones que mitiguen la problemática ambiental en Juanchaco, con miras a generar una mejora en su calidad de vida.

Posteriormente, la evaluación de las propuestas se realizó bajo criterios de impacto social, aceptación por parte de la comunidad, generación de beneficios económicos y ambientales y factibilidad desde los aspectos técnicos y de fabricación. De esta manera, se asegura éste como un proyecto deseable, viable y factible, acorde a la problemática ambiental y al desarrollo sostenible de la comunidad.

Materialización: por último, se probó la propuesta de diseño para hacer una validación en contexto con los usuarios reales. La experimentación y la iteración sobre la propuesta permitieron construir una solución a la medida, evaluando, por medio de observación y registro cuantificable, el cumplimiento de los objetivos propuestos. Después de permitir a los usuarios interactuar con el artefacto, se tomaron comentarios y opiniones por parte de las personas involucradas.

\section{Resultados}

Con el desarrollo de la primera etapa, se hizo evidente la problemática de basuras en Juanchaco, la que trae consecuencias ambientales, sociales, económicas y culturales, afectando la calidad de vida de los nativos de la isla.

De esta manera, se identificó que los residuos llegan a la isla de tres maneras: la primera corresponde a las corrientes oceánicas y vientos que concentran residuos plásticos, para formar islas que se desplazan a lo largo de la costa Pacífica. Según el artículo de Helen Briggs en la BBC news, estas concentraciones de basura pueden contener, por lo menos, 80.000 toneladas de plástico. Debido a la posición geográfica de Juanchaco, que se encuentra en un estrecho canal rocoso (Cioh.org.co, 2019), se genera un asentamiento sin salida de residuos plásticos proveniente de las corrientes marítimas mencionadas, ocasionando que se recojan hasta tres toneladas de basura en un fin de semana, dato comprobado en el trabajo de campo.

La segunda es el consumo eufórico de productos desechables en la isla y su mala disposición. Esto se debe a factores asociados a costos, comodidad y el difícil acceso a elementos básicos como el agua. Tercero, la falta de involucramiento de los nativos de Juanchaco con el manejo de las basuras acumuladas en la playa, lo que pone en riesgo al ecosistema.

Así, se evidenció que los espacios con fines de expresión artística y cultural, terminan como lugar de disposición final de los desechos, ya que en la isla no hay otro lugar en el que los residuos puedan permanecer mientras un barco de la armada nacional llega a recogerlos, para generar problemas de salubridad en el ambiente, como se evidencia en la figura 1. 


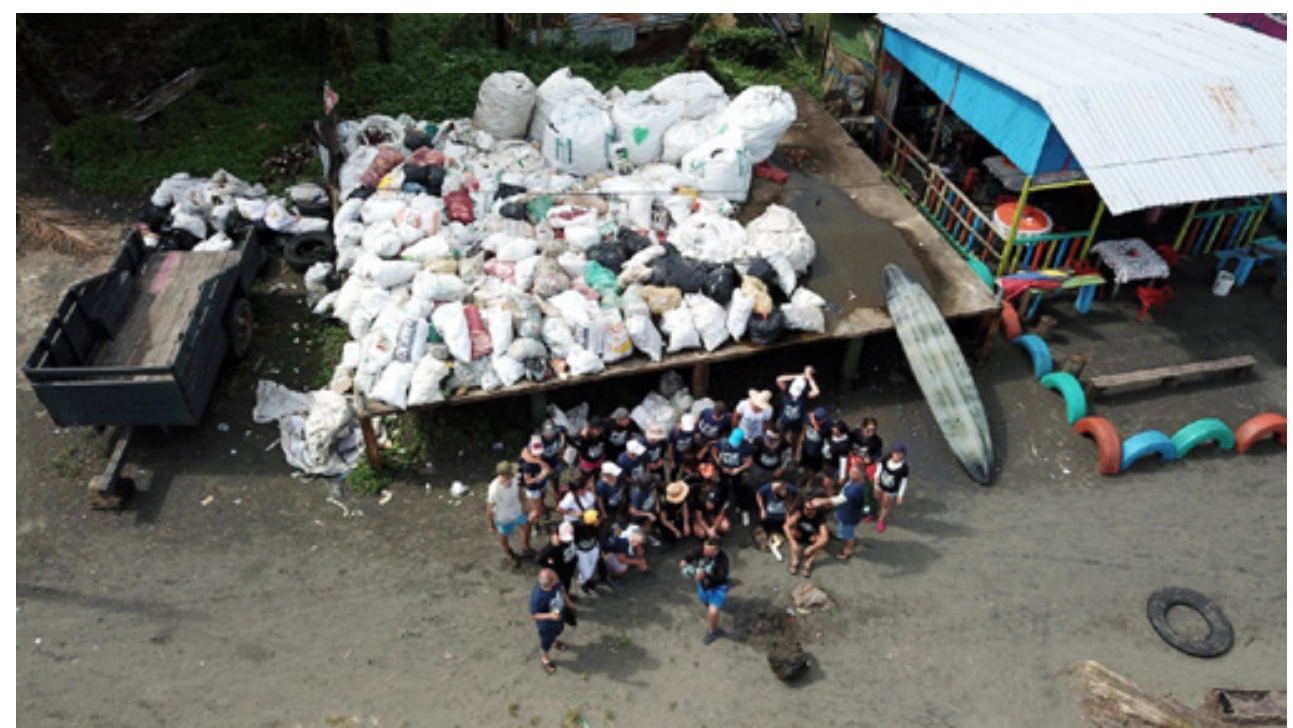

Figura 1. Tarima artística llena de basura en Juanchaco

Fuente: Trabajo de campo del semillero espacio $D+E+1,2019$.

Con la participación en una brigada de limpieza en conjunto con la fundación EcoPAZcifico, los integrantes del proyecto, nativos y voluntarios intervinieron un área de la playa de Juanchaco. La jornada de limpieza estuvo dividida en dos fases: en la mañana, el proceso de recolección de desechos plásticos, principalmente, Poliestireno Expandido (PET), PS y PEBD, de los cuales se recolectaron, aproximadamente, 950 kilos en una jornada de trabajo.

\section{- Etapa 1:}

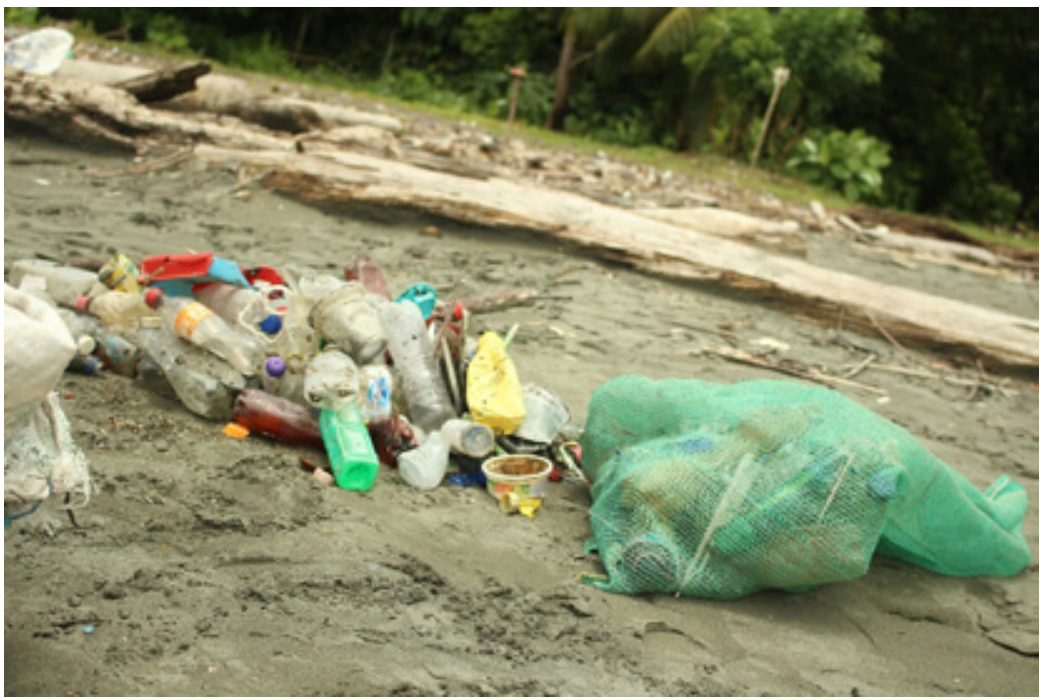

Figura 2. Sector de la playa a intervenir

Fuente: Trabajo de campo semillero espacio D+E+1, 2019. 


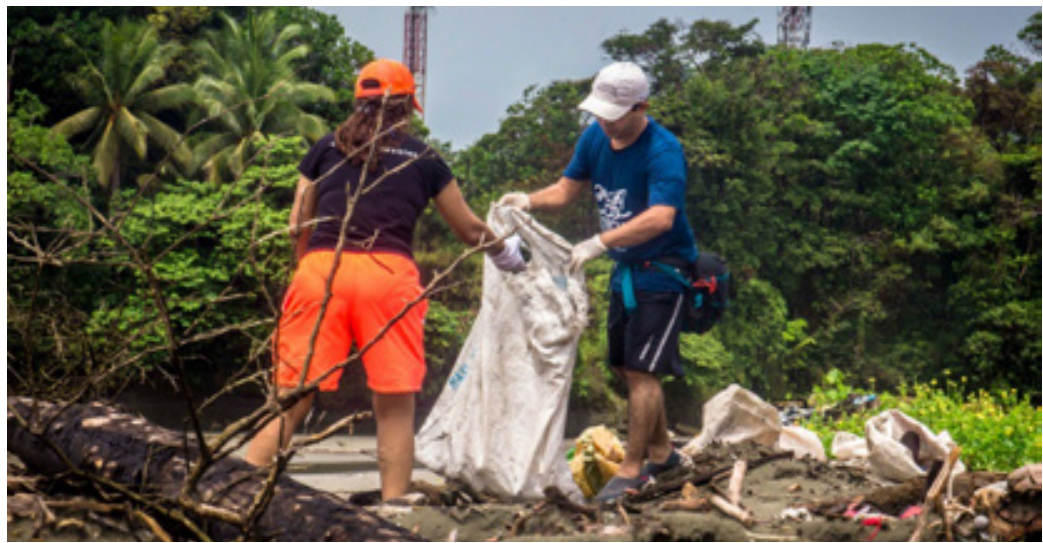

Figura 3. Recolección de residuos

Fuente: Trabajo de campo semillero espacio $D+E+1,2019$

Al finalizar la primera fase del proyecto, se encontró que los residuos encontrados son, en gran parte, derivados del plástico e, incluso, también se encontró que muchos miembros de la comunidad aportan a la problemática ambiental, debido a que tienen prácticas que atentan contra el cuidado de la playa. Esto se debe a que los habitantes de Juanchaco tienen como prioridad, la extracción de madera y la pesca artesanal, y dejan a un lado la limpieza de la playa, pues dicha actividad no es considerada como fuente de ingresos extra o de beneficios.

Luego, en la jornada de la tarde, se dedicó a la separación y limpieza del material recolectado para su disposición y transformación (véase figura 4). Esta última fase permitió resignificar el concepto de basura a Maparé, determinado por la organización como Material para revivir, utilizado en la realización de una composición artística con niños y personas de la comunidad que participaron en la brigada. El Maparé es la materia prima para el desarrollo artístico y cultural de la comunidad.

\section{- Etapa2:}

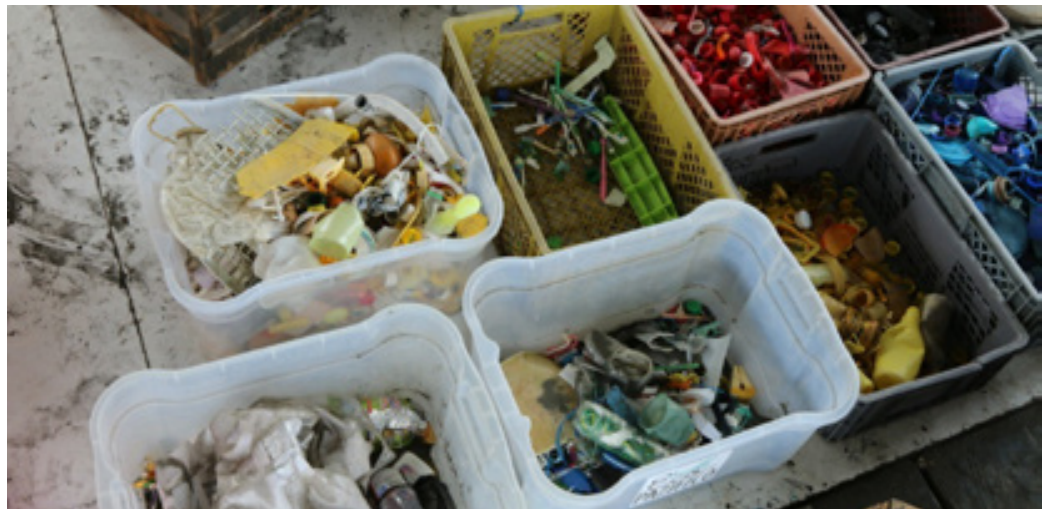

Figura 4. Separación y limpieza de Maparé

Fuente: Trabajo de campo semillero espacio $D+E+I, 2019$. 
Un ejemplo de lo que se puede llegar a crear con esta iniciativa, se puede ver reflejado en la pieza que se realizó con el material recolectado en la jornada de limpieza. En esencia, con la creación de esta pieza grafica se pretende resaltar la diversidad de fauna y el valor de la naturaleza por la que se destaca Juanchaco. Creada con material recolectado de su playa, esta pieza artística será parte de una exposición de arte en la ciudad de Cali, que tendrá como tema central el arte construido a partir de la recuperación de desechos (véase figura 5).

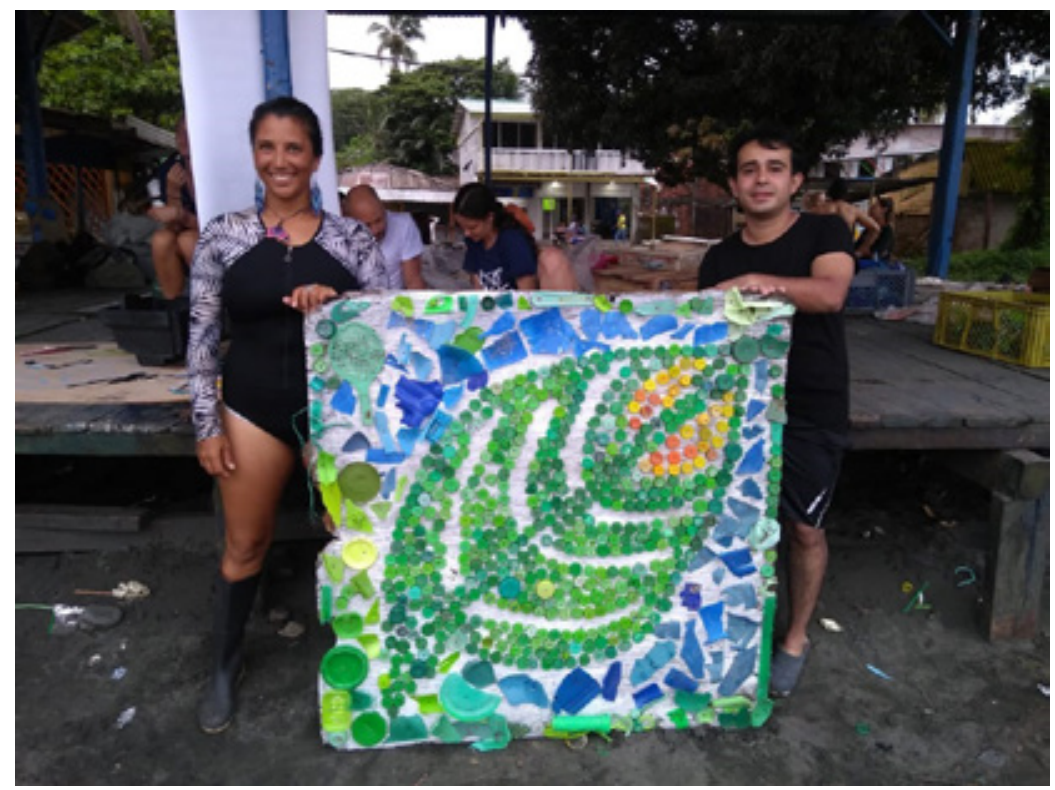

Figura 5. Pieza realizada a partir del Maparé

Fuente: Trabajo de campo semillero espacio $D+E+1,2019$.

De esta manera, el proyecto busca resignificar los desperdicios que se generan o llegan a la playa, por medio de un ciclo que permita expresar, de manera artística, las labores alrededor de la responsabilidad ambiental, de manera que se puedan convertiren fuentes alternativas de ingresos monetarios para las familias, con la generación de productos a base de plástico recuperado, que muestren y resalten aspectos culturales y sociales que resalten a la zona Pacífica y su gente.

Lo anterior, al permitir que la expresión artística se constituya en un motor desarrollo y visibilidad para la comunidad, con la transformación de los residuos plásticos y su integración en un ciclo que comprenda desde la recolección hasta la transformación de productos o piezas, combinando saberes de jóvenes con experiencia y vivencias de los adultos. Con la implementación de un sistema de aprendizaje gráfico, donde se ilustren diversas formas de hacer productos, a través de manuales gráficos y técnicas de construcción básicas que faciliten la expresión de un arte diferente.

De la misma forma, se quiere aprovechar la intervención de aquellos individuos de la comunidad que son reconocidos por ser la voz de cambio en los jóvenes, como promotores de nuevos hábitos de reciclaje y reutilización de desperdicios, para convertir a la juventud en monitores y gestores de cuidado y bienestar de la isla. 


\section{Referencias}

Cioh.org.co. (2019). Canales de acceso [Mensaje en sitio web]. Recuperado de https://www. cioh.org.co/derrotero/index.php

Guevara Montesinos, O. (17 de mayo de 2017). ¿Cómo realizar una transformación cultural en las organizaciones? [Mensaje en sitio web]. Recuperado de https://www.esan.edu. pe/conexion/actualidad/2017/05/17/como-realizar-una-transformacion-culturalen-las-organizaciones/

Molina Moreira, M.N. (julio-diciembre, 2017). Diagnóstico del manejo de residuos sólidos en el Parque Histórico Guayaquil. La Granja, 26(2), 84-105. Recuperado de https:// dialnet.unirioja.es/servlet/articulo?codigo $=6094543$

Parques Nacionales Naturales de Colombia. (18 de octubre de 2019). Parques Nacionales Naturales de Colombia. [Mensaje en sitio web]. Recuperado de http://www. parquesnacionales.gov.co/portal/es/ecoturismo/region-pacifico/parque-nacionalnatural-uramba-bahia-malaga/

Resultados de pobreza en 2018 por regiones y ciudades. (15 de julio de 2019) ¿Y dónde están los pobres de Colombia? [Mensaje en sitio web]. Recuperado de https://www. dinero.com/economia/articulo/resultados-de-pobreza-en-2018-por-regiones-yciudades/270554

Rodas, A. (22 de mayo de 2017). Buenaventura, el puerto del Pacífico condenado al olvido y la violencia [Mensaje en sitio web]. Recuperado de https://prensarural.org/spip/spip. php?article21540

Serrano, S. (9 de mayo de 2019). La caleña que lucha contra la contaminación con plástico en el Pacífico. El País. Recuperado de https://www.elpais.com.co/valle/la-calenaque-lucha-contra-la-contaminacion-con-plastico-en-el-pacifico.html

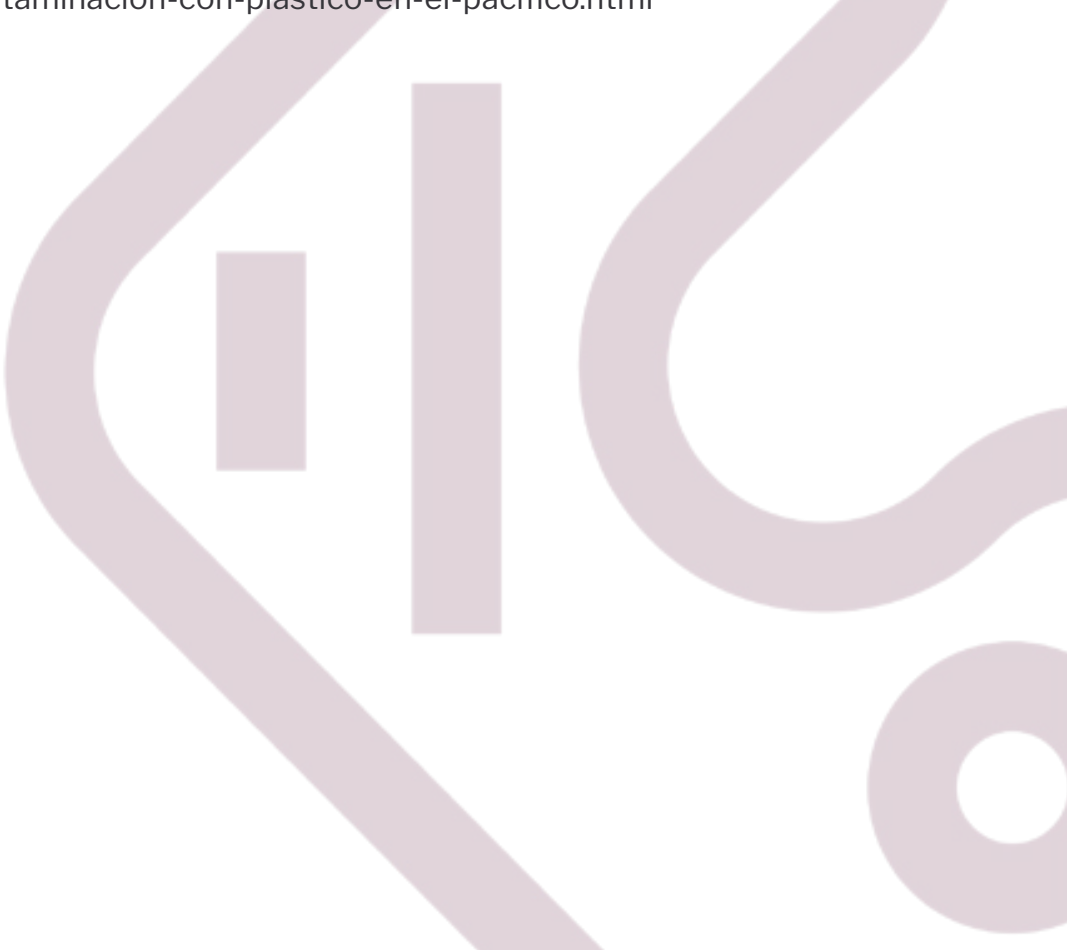

